\title{
Entrelacs
}

Cinéma et audiovisuel

\section{Cinéma : connaissances, croyances et idéologies}

Transformations du cinéma, transformations des spectateurs : ce que peut le cinéma

David Faroult

\section{(2) OpenEdition}

\section{Journals}

Édition électronique

URL : http://journals.openedition.org/entrelacs/57

DOI : $10.4000 /$ entrelacs. 57

ISSN : 2261-5482

Éditeur

Éditions Téraèdre

\section{Édition imprimée}

Date de publication : 1 novembre 2005

Pagination : 21-37

ISBN : 978-2-912868-70-1

ISSN : 1266-7188

Référence électronique

David Faroult, «Cinéma : connaissances, croyances et idéologies », Entrelacs [En ligne], 5 | 2005, mis en ligne le 01 août 2012, consulté le 01 mai 2019. URL : http://journals.openedition.org/entrelacs/57 DOI : 10.4000/entrelacs.57

Ce document a été généré automatiquement le 1 mai 2019.

Tous droits réservés 


\section{Cinéma : connaissances, croyances et idéologies}

Transformations du cinéma, transformations des spectateurs : ce que peut le cinéma

\section{David Faroult}

1 Des connaissances se désignant aujourd'hui comme "sciences » se trouveront peut-être demain ramenées au nombre des idéologies, cette opération s'accompagnant d'une explication (idéologique, politique, historique, économique, ...) de leur fugitive promotion au rang de science. Ainsi, aujourd'hui, chacun sait que l'église a longtemps censuré et réprimé les auteurs de systèmes héliocentriques (Galilée, Copernic, Kepler) : on sait que l'Église y voyait l'amorce d'une dangereuse mise en doute de ses dogmes. Il se peut donc que derrière des connaissances scientifiques, se cachent souvent des enjeux politiques, même quand le lien n'est pas immédiat. L'exemple de Galilée a ceci d'éloquent qu'on y perçoit bien en quoi un nouveau savoir peut se trouver menaçant pour des croyances sur lesquelles s'appuie le pouvoir (politique). Du coup, les croyances tenues à l'époque pour la seule vérité possible, sont aujourd'hui reconnues (par l'Église elle-même) comme d'anciennes croyances.

2 Les discours idéologiques revêtent le plus souvent les apparences du discours scientifique et n'en relèvent pas moins de présupposés qui les relient à des positions pratiques, en dernière instance : politiques ${ }^{1}$.

Comment distinguer une proposition idéologique d'une connaissance, y compris d'une connaissance «scientifique »? On peut bien sûr, de façon pragmatique, s'interroger sur l'éventuel intérêt qu'un individu peut avoir à répandre certaines représentations. Le problème s'épaissit dès lors que telle validation des connaissances (par exemple: l'empirisme) se trouve admise dans tel champ (par exemple: la médecine) et se trouve remise en question pour appréhender d'autres domaines. Quel raciste n'a pas invoqué l'argument empirique pour étayer les maux dont il accable ses cibles? («Je les connais moi ! », " Je les ai vu en Algérie/en Indochine/dans mon quartier/etc. ») 
faisaient même la guerre. Mais ils ne gardaient leur foi qu'à un seul. La foi, c'était leur engagement inconditionnel. $»^{2}$ Les croyances auxquelles nous adhérons ne se réduisent pas à nos convictions, nos engagements, et elles se présentent rarement comme étant en relation avec ces convictions. Ainsi, tel rationaliste qui se pensait à l'abri des superstitions, se réjouira qu'il ne lui soit rien arrivé de fâcheux après être passé sous une échelle : il y verra la preuve de la stupidité des superstitions. N'empêche qu'il y croit assez pour n'avoir pas pu se dispenser d'y penser ! C'est que, d'après Freud, si le conscient sait opérer une distinction entre la réalité et l'imaginaire, ces distinctions n'existent pas dans l'inconscient. Une représentation imaginaire ancrée dans l'inconscient d'un individu se révélera largement aussi efficace, pour influer sur son comportement, que tous les discours rationnels auxquels ce même individu prétendra adhérer (tous les analysants saisiront aisément ce que je veux dire...).

6 Il me semble qu'on peut néanmoins, avec Gérard Leblanc, établir une distinction entre deux types de constructions imaginaires : celles qui s'orientent vers l'évasion et celles qui s'orientent vers la résistance. Les modalités d'enfermement des individus dans les prisons ou en dehors, présentent sans doute beaucoup plus de similitude qu'il n'y parait au premier abord. En particulier, le recours à l'imaginaire répond dans ces deux modalités au même besoin de se maintenir (survivre) malgré les coercitions directes ou indirectes, explicites ou insidieuses, auxquelles on est soumis. Cet imaginaire s'oriente vers l'évasion, c'est-à-dire l'illusion auto-produite d'une vie meilleure en un ailleurs, ou vers la résistance, c'est-à-dire, intégrant les conditions réelles de la servitude, rêvant ses transformations libératrices. Dans l'imaginaire d'évasion, l'autonomie entretenue entre réalité et imaginaire, est propre à assurer la reproduction de la réalité telle qu'elle est. Cet imaginaire n'incite pas le sujet à lutter contre les conditions réellement existantes qui lui sont imposées ${ }^{3}$.

7 L'imaginaire de résistance, au contraire, se nourrissant des conditions de la réalité, qu'il prend en compte sans atrophier sa fonction fantaisiste, est propre à armer davantage le sujet pour faire face aux situations réelles. Le sujet ne se rêve pas en un utopique « ailleurs » (étymologiquement, l'utopie n'a pas de lieu), mais se conçoit ici, autrement. Il va de soi que cette distinction entre deux types d'imaginaires n'exclut absolument pas que les deux s'affrontent au sein du même individu, soient sollicitées successivement ou simultanément.

8 Dans leurs dispositifs, le cinéma, comme le théâtre, instaurent une illusion à laquelle le spectateur se prête. Mais ce serait une erreur de croire que cette illusion nous renverrait à la réalité, qu'il s'agirait d'une « illusion de réalité » des choses représentées. A ce titre, ce n'est pas une illusion comme les autres : il ne s'agit pas d'être trompé ou non, de même que l'identification du spectateur au personnage est différente du processus pathologique de l'identification hystérique. Dans le processus de l'illusion cinématographique ou théâtrale, le problème de la distinction entre le réel et l'imaginaire n'est pas posé. Comme le suggère Octave Mannoni, nous sommes plutôt les complices d'un dispositif qui pourrait, idéalement, duper certains individus : on se plaît à raconter que des spectateurs « primitifs » ont pu être terrorisés par l'arrivée d'un train en gare de la Ciotat, comme si, eux, étaient réellement en proie à l'illusion du dispositif cinématographique. Ceci nous 
autorise à faire semblant de croire à la réalité représentée, mais sur un mode ludique ou symbolique : je sais que Belmondo ne meurt pas VRAIMENT à la fin du film, mais ça ne m'empêche pas de m'émouvoir de la mort de son personnage (que je sais être fictif) »4.

9 Tout film, dans quelque secteur de l'audiovisuel qu'il apparaisse (cinéma, télévision, audiovisuel d'entreprise, etc.) entretient un rapport avec la réalité en tant qu'il en constitue une représentation, et ce quel que soit le genre auquel il se rattache (documentaire, fiction, ou même science-fiction) ${ }^{5}$.

10 Un film, quel que soit son genre, sollicite des représentations (parmi lesquelles certaines sont déjà catégorisées comme " connaissances ») déjà existantes dans les cerveaux des spectateurs. Un film de science-fiction qui s'annonce comme se déroulant «il y a bien longtemps, dans une galaxie très lointaine" sollicite quelques notions d'astronomie, par exemple (parmi les plus élémentaires, soit). Tel documentaire sur tel pays d'Afrique, suppose déjà connu que l'Afrique est un continent situé au sud de l'Europe (notion élémentaire de géographie). Mais avec ces connaissances sollicitées, toutes sortes de représentations acquises se joignent comme «associées» aux connaissances: des « clichés» et autres stéréotypes, et toutes les représentations plus ou moins conscientes dont chaque spectateur, dans sa singularité spécifique, peut disposer sur un domaine déterminé.

11 Or, à toutes ces représentations acquises, le film va changer quelque chose (le film y change forcément quelque chose). Soit il va renforcer les représentations les plus répandues, déjà ancrées : renforcer les stéréotypes intégrés par le spectateur. Notons que pour qu'une représentation soit particulièrement répandue parmi les spectateurs, il faut qu'elle appartienne à un corpus des représentations dominantes: appelons cela "l'idéologie dominante». Le film peut encore ajouter quelque chose aux représentations déjà ancrées. Mais le film peut aussi fragiliser, mettre en doute ou transformer les représentations qu'il sollicite. Je me souviens qu'enfant, j'avais été très surpris devant un film de Jean Rouch montrant une ville africaine : avant ce film je croyais que les Africains vivaient tous dans des huttes en paille! (Je ne raconte pas cela pour faire étalage de mes souvenirs d'enfance, mais parce que, enfant, je ne m'étais sûrement pas mis tout seul cette idée, fort coloniale, en tête...).

12 Tout film change donc peu ou prou l'état de nos représentations de la réalité et de connaissances en particulier : il produit des effets de connaissance. Le film change forcément quelque chose à l'état de nos représentation: on en sait donc " un peu plus », ou « un peu mieux » sur ce qu'on connaissait déjà, ou sur ce qu'on ne connaissait pas encore avant le film. C'est cet effet de transformation inévitable de nos représentations qu'on pourrait appeler "effet de connaissance», puisque c'est de nos connaissances, de ce type particulier de représentations qu'il s'agit.

13 Chaque scénario, en ordonnant la succession des effets de connaissance d'une certaine façon, met en œuvre une conception philosophique : sa conception épistémologique de l'acquisition des connaissances. C'est-à-dire que chaque scénario, quelque conscience qu'en aient ses auteurs, met en œuvre une "théorie de la connaissance». En cela, Cinéthique avait raison d'affirmer : "Il est possible de saisir qu'en tout 'processus esthétique' travaille une théorie de la connaissance $"\left(\mathrm{n}^{\circ} 13-14, \mathrm{p} .54\right)$.

14 On entend par "théorie de la connaissance $»^{6}$, la conception épistémologique des opérations en jeu dans l'acquisition des connaissances. On pourra interroger cette théorie sur quelques points, qu'on peut juger décisifs : quelle est la place de la pratique dans une 
théorie donnée? Comment échappe-t-elle à l'écueil empirique en ménageant un «moment théorique » distinct du moment pratique ? Etc.

C'est à l'aune de ce type de questionnements qu'on pourra caractériser une théorie de la connaissance et éventuellement la rattacher à des doctrines philosophiques déjà constituées. En d'autres termes: chaque film procède d'une certaine conception de l'acquisition des connaissances (sa «théorie de la connaissance »), et cette conception le rattache à des conceptions philosophiques plus générales, dans la mesure où ces «théories de la connaissance » sont l'expression de présupposés philosophiques. On peut risquer une re-formulation simplifiée de la thèse de Cinéthique: en tout processus esthétique travaillent des présupposés repérables.

\section{Chaque élément en jeu dans la conception d'un film se ramène à une opération de scénarisation}

Le problème se complexifie d'autant plus qu'on admettra que chacune des opérations liées à la confection du film peut se rapporter à une opération de «scénarisation ». En effet, s'il est entendu que le "canevas » commun à une équipe de cinéma est ce qu'on désigne comme un « scénario de tournage » (quel que soit son degré de précision : simple synopsis, dialogues, découpage, story-board...), il n'en reste pas moins que chaque contribution apporte des éléments concrets qui « raconte » davantage, qui «scénarise ». Le physique d'un comédien scénarise son personnage d'une certaine façon, sa voix également, ou différemment : on dit d'ailleurs parfois d'un comédien qu'il est utilisé «à contre-emploi » dans un rôle, ce qui suppose non seulement qu'on associe son physique et sa voix à certains personnages, mais encore qu'on imaginait quelqu'un d'autre (ayant d'autres caractéristiques physiques, vocales ou autres, plus ou moins clairement formulables) pour incarner ce personnage-là. Cet exemple est un symptôme parmi les innombrables, de l'intégration diffuse, non-dite, mais enracinée, d'une certaine " norme " de «ce qu'il faut faire». Cette "habitude» (une "seconde nature», dit-on) est la meilleure des garantes de la reproduction du cinéma dominant.

17 Ce qui est vrai pour l'acteur l'est aussi de la lumière, du son : autant d'opérations qui à chaque instant de la concrétisation du film le scénarisent un peu plus précisément. Or, dans chaque opération de scénarisation, une certaine conception du monde (et de cette opération) est mise en œuvre. Ces conceptions, bien que souvent non-formulées et même inconscientes, n'en sont pas moins repérables et caractérisables. C'est en cela qu'on peut les identifier à des idéologies pratiques. On voit bien ainsi que « l'idéologie $»^{7}$ d'un film n'est pas réductible à celle qu'il affiche, mais est un réseau d'idéologies pratiques mises en œuvre dans sa confection, et qui peuvent se trouver contradictoires. Ce qui peut se repérer comme idéologies dans un film se manifeste donc comme une lutte de tendances, au sein de laquelle il s'agira de démêler lesquelles sont principales et laquelle (ou lesquelles) domine(nt).

\section{Les stéréotypes: une matière première}

18 Des films produisent des effets de connaissance: ils reconduisent sempiternellement les mêmes scénarios (ex. : « un marin a une femme dans chaque port... ») qui une fois qu'ils sont largement intégrés, pénétrés, deviennent des «clichés 》 (mais n'en demeurent pas 
moins des scénarios) qui peuvent à leur tour être sollicités sans qu'un développement soit nécessaire. (ex : un personnage de marin $=>$ il a donc une femme dans chaque port...). structures-fonctionnements que l'on désigne respectivement par idéologie et inconscient est de dissimuler leur propre existence à l'intérieur même de leur fonctionnement en produisant un tissu d'évidences "subjectives", ce dernier adjectif étant à entendre non pas comme "qui affectent le sujet », mais « dans lesquelles se constitue le sujet »... » L'inconscient, l'idéologie sont donc des processus actifs, c'est à ce titre qu'il peuvent constituer l'individu en sujet. Cette théorie, difficile à saisir, présente l'intérêt d'expliquer le fonctionnement des idéologies comme processus dynamique. Elle rompt en cela avec une conception d'idéologies qui seraient constituées dans une sphère supérieure (superstructure), et auxquels les individus-sujets choisiraient en leur âme et conscience d'adhérer ou pas. Non: le processus d'appropriation des idéologies 1) permet à l'individu de se constituer comme sujet ; $2^{\circ}$ ) permet à l'idéologie de se répandre, par ce même mouvement ; $3^{\circ}$ ) permet au sujet de ne pas parler en son nom et de protéger ainsi son Moi en s'appuyant sur les clichés et stéréotypes déjà constitués. Du reste, l'inconscient n'établit pas de discrimination entre ce qu'il serait légitime ou illégitime de penser. Dans cette région de l'inconscient peuvent donc coexister des conceptions inconciliables, mais qui cependant opèrent ensemble sur le sujet qui les héberge.

Il n'est pas indifférent qu'un film se présente comme appartenant au genre, codifié, du documentaire ou à celui de la fiction. L'un et l'autre n'appellent pas le même degré de vigilance du spectateur, la conscience qu'il se fait de la relation du film avec la réalité n'est d'emblée pas la même entre les deux. Le film de fiction dispose à ce titre d'une efficience multiple par la neutralisation de la vigilance du sujet :

- le genre fiction appelle d'emblée une vigilance réduite,

- constitué de représentations imaginaires, la fiction peut s’appuyer sur des croyances (conscientes/inconscientes) qui n'ont pas besoin d'être rationalisées, qui n'appellent donc 
pas de légitimation ou justification : le processus d'appropriation de l'idéologie du film, s'opère donc comme une intériorisation par le sujet de croyances dont le caractère non rationnel rend vaine toute vigilance ;

- la réception du film a lieu dans le temps de loisirs, c'est-à-dire le temps de reproduction de la force de travail, temps pendant lequel, sous l'effet de la fatigue, la vigilance est déjà réduite.

Le spectateur dispose donc pour tout personnage ou tout sujet de film, d'un arsenal de scénarios disponibles, tous préconstruits, correspondant à des représentations sociales toujours-déjà ancrées. C'est lui qui sollicite ces scénarios à la demande et bénéficie ainsi de l'illusion de mettre en œuvre sa propre «culture »et son «libre arbitre » dans le processus de réception du film. Cette opération de sollicitation de "déjà-connus " s'inscrit dans des localisations diverses, dont on réduira pour l'instant la topologie à : des savoirs conscients, inconscients, ou préconscients du spectateur. Pour être localisés différemment, ces « savoirs » idéologiques n'en sont pas moins efficients.

\section{Pas d'Autre?}

Reconduire indéfiniment les mêmes scénarios, sans les questionner, les pervertir, les déconstruire, évite finalement toute possibilité de transformation des individus par une confrontation à l'altérité, à l'Autre. Une Rencontre n'est possible, au sens où elle est potentiellement productive, qu'à la condition que l'on soit prêt à subir une transformation réelle. Les films font partie du réel : pourquoi les rencontres avec les films seraient-elles moins importantes que celles avec les individus?

$\mathrm{Du}$ point de vue de la reproduction de l'Ordre (du désordre?) social existant, la contradiction à résoudre est : « Comment satisfaire un besoin d'évasion produit par les tensions de la vie quotidienne (besoin de "faire un break", une "pause», besoin d'évasion, ...besoin de reproduction de la force de travail) en évitant que la satisfaction de ce besoin ne favorise une transformation des sujets dans un sens qui les conduirait à contester l'Ordre: l'organisation de la société ?».

Ainsi, le temps des vacances s'organise-t-il autour d'une pratique du tourisme qui (tout en étant un secteur économique juteux, à l'instar du cinéma) est codifiée, scénarisée autour d'un principe : simuler la rencontre de l'Autre tout en évitant qu'elle advienne. C'est pourquoi on va loin (d'autres pays, d'autres continents), on visite d'autres cultures, mais seulement dans les musées et les simulations de traditions locales typiques. (Voir Marrakech, c'est voir son souk et y marchander, voir Athènes c'est voir le Parthénon et commander ses souvlakis en grec, grâces aux guides de conversations touristiques qui n'indiquent presque que des situations d'achat). En tout cas on évite que puisse s'instaurer un dialogue productif entre des gens issus de pays et de cultures différentes, sans doute par crainte que ce dialogue ne déstabilise l'ordre des hôtes tant que celui des visiteurs, par un effet qui ressemblerait à celui des Lettres Persanes. Un regard "persan » (perçant !), « candide » sur notre société nous serait donné sur notre propre organisation sociale, par la surprise que provoquerait, chez notre interlocuteur, l'évocation d'une évidence admise pour nous, dans notre pays. C'est un des effets possibles d'une confrontation avec l'Autre, qu'il convient à tout prix d'éviter pour préserver telle qu'elle est l'organisation de la société. 

est 'entre', c'est à dire protégé. (...) Mais ils [le «public normal»] sont devenus des touristes (consommateurs de voyages) et ils n'attendent plus du cinéma qu'il leur 'donne' le frisson de l'exotisme, ni du film qu'il les y mène à son rythme (lent) ». (L'exercice a été profitable, Monsieur, P.O.L., Paris, 1993, p. 23)

31 Mais cet Autre à rencontrer, peut aussi se loger à l'intérieur de l'individu : rencontrer l'autre, c'est aussi rencontrer l'autre en soi-même.

32 Ne pourrait-on voir en Justine de Sade, un livre qui lit en nous? Voilà le scandale de Sade : il ne répugne pas d'emblée. Au début, il peut amuser, émoustiller, exciter même. Puis les images sollicitées/construites dans l'imagination du lecteur s'écartent des pratiques socialement acceptables, puis insensiblement: des pratiques humainement acceptables. Mais où est la limite? C'est en ce point que le livre lit en nous : « Tu as accepté jusque là. (Je t'ai même surpris excité en ce point ci.) " nous dit le livre, ne nous laissant comme seule voie de dénégation, que le refoulement de nos propres fantasmes. Sade lit en nous. Et c'est cela qui est inacceptable.

\section{Des musiques nous écoutent}

Cecil Taylor, est un pianiste de Free Jazz.

Le $1^{\mathrm{er}}$ juillet 2000, une salle de concert à la Villette, sous l'ancienne grande halle des bouchers. Une salle de concert, pleine, est plongée dans l'obscurité totale. Après un instant de silence, on entend des borborygmes proférés dans un micro. L'homme qui profère ces sons gutturaux (inconvenants, obscènes ?) s'approche lentement du piano. On ne le voit pas, mais on le sent qu'il s'en approche, ou alors on le sait, on s'en doute... Il commence à «jouer» du piano. Mais ce jeu, ne ressemble pas à ce qu'on a l'habitude de désigner comme de la musique. L'audience est attentiste. La lumière monte, très lentement, elle restera à un niveau faible, pendant tout le concert, comme entre chien et loup. Comme une invitation à fermer les yeux, au lieu de les ouvrir. On peut même s'assoupir, si l'on veut. Cecil Taylor joue. Il ne s'interrompt pas. "Il se moque du monde! C'est n'importe quoi!» Le chaos apparent dissimule au premier abord la profonde cohérence de la musique. Alors l'auditeur se rebelle : «C'est n'importe quoi!». Mais contre quoi, au juste, proteste-t-il ? La musique a commencé à faire son travail. Elle stimule l'imagination, et les états sensibles que l'interprète sollicite pour improviser, l'auditeur commence à les éprouver lui-même, mais différents. C'est sa propre imagination, sa propre mémoire et sa propre sensibilité qui ont été sollicités par la musique. La musique l'écoute, à l'intérieur. 

manifestent, à la faveur de digressions du récit dont ce cinéaste a le secret, leur attachement au monde naturel. La petite fille de Mater Dolorosa, doit nous attendrir en se plongeant dans un bocal à poissons. Cet épisode est non seulement repris dans le remake parlant (également réalisé par Gance), mais il est en plus augmenté d'une réplique de la petite fille qui avant sa baignade confesse «J'aimerais bien être un poisson! ». Dans La Roue, le personnage de Sisif (sic.), est en harmonie avec le monde qui l'environne, en particulier avec sa locomotive, qu'il aime comme une femme. Cette harmonie ne sera que partiellement altérée par son handicap de cécité qui ne l'empêche pas de s'orienter sur les chemins neigeux d'une montagne, aux précipices menaçants. Napoléon, lui-même, dans Napoléon vu par Abel Gance, saura traverser une tempête comme si lui-même faisait partie de son tumulte. Napoléon est au milieu de la tempête, à bord d'un frêle esquif sur lequel, en guise de voile, il a hissé un drapeau français. La tempête hâte son départ forcé et précipité de la Corse, où il est devenu indésirable. La tempête lui est donc favorable en un sens, c'est en tout cas ainsi que Gance nous la fait éprouver. plus privilégiées, induisant, par métonymie, une relation forte avec la Nature (avec l'ensemble de la création) ${ }^{9}$ : l'aiglon de Napoléon, les oiseaux avec lesquels s'entretient Cyrano, juste avant sa rencontre avec D'Artagnan, les trois cigognes du blason du Capitaine Fracasse, la pie voleuse de Jérôme Perreau, héros des barricades (qui jouera un rôle décisif dans la victoire de la Fronde), c'est aussi par le silence des oiseaux que Beethoven traversera l'épreuve tragique de sa première crise de surdité... Dans des proportions variables selon le degré de liberté dont disposait Gance pour concrétiser ces différents films, chacune des séquences évoquées constitue une digression poétique, sollicitant des effets plus ou moins élaborés, pour faire partager par le spectateur la complicité d'un 
personnage avec la nature, sa relation poétique au monde trouve une forme imagée et sonorisée. Surimpressions et multiplication de l'écran dans Napoléon, a-synchronisme radical dans Un grand amour de Beethoven, mobilité de la caméra et vivacité du montage dans Cyrano et d'Artagnan, ou très sobrement : plan séquence fixe dans la version muette de Mater Dolorosa... La formalisation d'une relation sensible, poétique au monde exige l'extension des moyens techniques disponibles et stimule les innovations et inventions de Gance. Nouveautés techniques qui doivent assurer un renouvellement des sensations éprouvées par le spectateur. Il s'agit de représenter cinématographiquement la pensée : une extension des techniques disponibles s'impose donc en fonction de ce projet de représentation, et non parce que de nouvelles techniques seraient apparues. Chez Gance, le projet de représentation précède et détermine les innovations techniques et non l'inverse.

Selon son propre aveu, il s'agissait pour Gance dès ses premiers films muets, de rendre visible ce que pensent et éprouvent les personnages. Il s'agit donc d'étendre les frontières $\mathrm{du}$ visible, du sensible (avec l'arrivée du son dans les années 30). Rendre sensible les personnages de leur intérieur, faire accéder le spectateur à une nouvelle connaissance sensible qui lui était inaccessible jusque là. Pour cela, il se comprend aisément que toutes les innovations techniques qui peuvent étendre le cinéma au-delà de son seul écran muet sont les bienvenues. On commence donc par ouvrir la possibilité d'un débordement de l'écran en lui en adjoignant deux autres à gauche et à droite, qui peuvent tripler la largeur de l'image ou bien proposer deux images, différentes de celle du centre, mais qui en sont le contrepoint. Ainsi, au cinéma qui ne disposait que du montage vertical successif, Gance ajoute son invention : le montage horizontal simultané (la polyvision ou triple écran). Dès l'apparition du son, il est patent que Gance n'accueille pas les dernières technologies, inventées par lui ou par d'autres, pour renforcer les possibilités naturalistes du cinéma (ajouter le son qui manquait à l'image), mais au contraire pour élargir le champ des connaissances sensibles accessibles par cet art. De même que dans ses films muets, Gance multipliait les usages du plan subjectif, du subjectif psychologique (le plan qui montre à quoi pense le personnage, avec ou sans surimpression), de même l'avènement du son permettra un recours au son subjectif (asynchrone), qui nous fait accéder à la pensée du personnage, sans cesser de le voir (c'est le cas dans la séquence de la surdité d'Un grand amour de Beethoven, les dilemmes des personnages de Mater Dolorosa parlant, et de façon plus rare mais non moins remarquable dans la Fin du monde, son premier film sonore et parlant). Dans Un grand amour de Beethoven, par exemple, on voit un gros plan du compositeur et on entend des sons (d'oiseaux, de ruisseaux, d'enfants...) dont Ludwig sait qu'il sera privé, maintenant qu'il est sourd.

\section{Des œuvres. Certaines, rares, nous regardent, nous spectateurs}

41 A supposer que des films puissent nous regarder. Prenons Méditerranée de Jean-Daniel Pollet, par exemple. Entre les pleins du film, le spectateur s'abîme. Les pleins : texte (Sollers), musique (Antoine Duhamel), images. Le chevauchement des trois éléments promet un sens (charge émotive forte de la musique, aspects perpétuellement introductif et grandiloquent du texte, aspect monumental et ancestral des sites et des grands espaces à l'image). Ce sens se dérobe sans cesse, toujours promis, jamais accessible, jamais lisible. Le spectateur ne peut recourir qu'à lui-même pour remplir l'abîme, la béance, la dérobade du sens promis par le film. Et le voilà, le spectateur (vous, moi...), regardé par le film. 
Qu'a-t-on à en dire, qu'y voit-on, sent-on ? Le film nous offre une monumentale promesse, et nous renvoie à nous même pour la tenir. Peut-on imaginer plus grande confiance placée dans le spectateur : le voilà (enfin !) mis sur un pied d'égalité avec le cinéaste ! S'il est un cinéma démocratique : le voilà ! Vois-là, un film qui te vois. Y es-tu prêt ? » Qu'estce que vous voulez que je fasse de toute cette liberté?» spectateur devra prendre en charge et assumer toute production de sens à partir de la matière qui lui est soumise et dont l'organisation lui échappe. Maintenant mis sur un pied d'égalité avec le cinéaste ( l'anti-Hitchcock»!), le spectateur peut se débattre (= débattre en lui-même ?) avec le film, comme dans la vie on se trouve parfois, face à des individus, à la rencontre d'un Autre.

Voici la promesse du film qui soudain prend une autre mesure : irréductible à un simple refus du sens qui la ferait ressembler à un appel à la phraséologie des théories de la modernité, le film rompt avec le cinéma de l'éternel retour du même (même scénario, cent fois reconduit, même silhouettes et même visages des vedettes...). Méditerranée nous invite à rencontrer l'Autre, et cette rencontre a lieu en nous: le film nous regarde faire. Méditerranée, objet d'une censure économique prolongée, réalisé en 1963, n'a connu de discrète sortie en salle qu'au bout de plusieurs années. "Le film regarde le spectateur réel en ce point où le cinéaste se sent regardé par ce qu'il filme. $»^{10}$ «Du haut de ces pyramides, quarante siècles nous contemplent!» disait, paraît-il Napoléon. Pollet se sentant, peut-être, contemplé par les pyramides, nous conduit, en ce point à être regardés par son film.

On trouve chez Jean-Daniel Pollet une invitation à questionner notre propre regard sur les lépreux, comme si son film L'Ordre, nous regardait avec le regard des lépreux? Retournement inattendu d'un film qui se présentait comme un documentaire sur la lèpre et plus particulièrement l'île de Spinalonga en Grèce, où les lépreux furent longtemps reclus. Ce film étant qui plus est réalisé sur la commande des laboratoires pharmaceutiques Sandoz qui espéraient une promotion de leur dernier traitement. Le même Pollet a réalisé plus récemment à partir de poèmes de Francis Ponge, un film qui nous regarde, nous « otages du monde muet »avec les « yeux »de ce monde : celui de nos propres objets. C'est Dieu sait quoi.

47 Voir ces films nous fait échapper à notre condition de consommateur d'évasion, à laquelle se réduit si souvent la situation de spectateur, pour nous inviter à des expériences humaines rendues possibles par les techniques du cinéma. Et finalement à quoi bon cette invention si elle ne se fixe pareille ambition. Mais, l'usage veut que les spectateurs parlent en terme de plaisir ou déplaisir. Soit, c'est de bonne guerre : une place de cinéma coûte presque $50 \mathrm{~F}$, alors pourquoi pas? Le film vous aura déplu? C'est le risque encouru lors d'une rencontre. Ne méritait-elle pas pour autant d'être tentée? Le film vous aura plu? Alors le mot est faible : vous aurez résonné (et raisonné) avec lui, vibré. Eprouvant la même sensation troublante et forte que lorsqu'une nouvelle rencontre inaugure une amitié, un amour, une complicité... Le cinéma est ici utilisé à la mesure de ce qu'il peut : produire des connaissances nouvelles et même plus: produire un nouveau mode sensible d'acquisition de connaissances.

La démarche de Jean-Daniel Pollet s'appuie sur une posture contemplative (et qu'importe que cette attitude contemplative ne soit pas très « à la mode » dans une époque où l'on est supposé s'émerveiller des vertiges de la vitesse.... ${ }^{11}$. Le temps consacré à regarder doit faire évoluer le regard posé sur les choses. Dans Dieu sait quoi, film autour de textes de 
Francis Ponge, Pollet prolonge notre observation d'objets évoqués par les textes (on sait que la poésie de Ponge repose largement sur la description verbale des objets, la matérialité des signifiants important tout autant que les signifiés mis en jeu dans la description). Même en tournant autour d'un objet par un travelling circulaire, même en exécutant plusieurs fois cette rotation, même en éprouvant les limites photographiques de la pellicule (en tournant le même plan à diverses heures du jour, en diverses saisons de l'année), même en saturant sa visibilité et sa représentation, un objet ne saurait être épuisé. Pourtant, on croyait que les premières secondes de sa première apparition auraient suffit à l'identifier, l'objet (d'habitude au cinéma, un insert sur un objet n'a pas besoin de plus de quelques secondes pour être identifié : voyez les gros plans d'objets chez Hitchcock. Les clés et ciseaux du Crime était presque parfait, bouteilles des Enchaînés, etc.). Dans Dieu sait quoi, on s'accordera le temps de la contemplation; son épuisement impossible devenant peu à peu l'objet du film.

La représentation elle-même est désignée comme inépuisable dans Ceux d'en face, le dernier film de Pollet, où l'agencement de photographies dans un ordre «juste » est un des principaux enjeux dramatiques du film. L'impossibilité de réduire les photographies au premier effet qu'elles produisent les vident puis les re-remplissent d'un faisceau de sens possibles, et ces divers mouvements de leur lecture se saisissent au gré de la durée et de la répétition de la contemplation des photos. Le cinéaste, veille d'ailleurs le plus souvent à ce que son cadre ne morde pas sur celui de la photo qu'il nous montre : nous devons en voir les limites. Finalement, inépuisables malgré leurs limites visibles, les photos frappent par leur étrangeté (elles nous sont étrangères) et cet Autre nous regarde les regarder, et nous regarde cesser de l'étiqueter (réflexe du premier regard:on reconnaît). «La poésie porte en elle la nécessité d'une seconde vue. Voir en poésie (...) c'est revoir ce qu'on a déjà $v u »^{12}$ Débarrassée des évidences supposées, qui s'imposent dès qu'on a nommé les choses, l'attitude poétique propose de les re-voir en instaurant avec elles une relation pré-langagière, ou plutôt post-langagière. Si la poésie consiste à «...pour apercevoir l'immédiat il ne faut pas réfléchir, ce qui implique qu'est déjà au travail en nous le parti pris du langage, mais se taire, plutôt, ouvrant grand les yeux. Et, constatant qu'il y a un monde, essayer, avec des mots qui s'interrogent sur leurs limites, d'appréhender les traits spécifiques de ce qui va se perdre du monde dès qu'on va parler de ce qu'on y voit. ${ }^{13}$ Les photos filmées par Pollet ne le sont-elles pas avec des cadres qui « s'interrogent sur leurs limites»? Quant aux textes qui hantent la bande-son pendant la contemplation des photos, il me semble que s'ils instaurent effectivement une interaction forte avec les images lorsqu'on les écoute attentivement, ils ont tout autant une fonction de contrepoint sonore (en tant que matériel sonore), au même titre que la musique d'Antoine Duhamel.

\section{Des possibilités didactiques du cinéma}

Je vais m'attarder sur quelques films : on aurait pu en choisir beaucoup d'autres. Ces films entretiennent avec des connaissances acquises « à l'extérieur » du cinéma un rapport de transmission. Je pense à une célèbre séquence du film d'Henri Verneuil I comme Icare... : celle où sont exposées les expériences de Stanley Milgram sur la soumission à l'autorité, mais aussi à une séquence moins célèbre (car le film l'est moins) d'un autre film du même : Le Serpent. 
51 Dans I comme Icare..., le procureur Volnay enquête sur l'assassinat du président Jarry (il s'agit de l'histoire de l'assassinat de J.F. Kennedy) un individu isolé est réputé être l'assassin du président. Cet individu a participé quelques temps auparavant à une expérience de psychologie sociale: c'est à ce titre que le procureur Volnay (Yves Montand) s'intéresse à cette expérience. On le conduit pour y assister dans une pièce surplombant la salle d'expérience, équipée de miroirs sans teint : il peut voir sans être vu. Dans la salle d'expérience, deux professeurs en blouse blanche reçoivent deux candidats : l'un sera le moniteur et l'autre l'élève dans une expérience sur la mémoire. Le moniteur lit une liste de mot auxquels sont associés des adjectifs, l'élève devra les mémoriser et restituer le bon adjectif au moniteur qui lui donne le mot. A chaque fois que l'élève se trompera, le moniteur devra lui infliger une punition : une décharge électrique. Il dispose pour cela d'un pupitre (avec des curseurs gradués de 15 en 15 volts de 0 à 450 ) relié à une chaise électrique qui accueille l'élève.

$\mathrm{Au}$ fil des erreurs de l'élève, les punitions augmentent : les professeurs prétendent étudier l'influence de la punition sur la mémoire. En réalité, l'élève est un de leurs complices, ils étudient la soumission à l'autorité du moniteur. Jusqu'où va-t-il torturer une victime innocente qui ne lui a rien fait, uniquement parce qu'une autorité supérieure le lui a demandé.

53 Voilà, brièvement résumé, le principe des expériences de Milgram, telles qu'elles sont rapportées dans le film, c'est-à-dire très fidèlement au livre dans lequel Milgram rapporte ses travaux. Le film ne change à peu près rien aux expériences réelles, mais ce peu nous intéresse : le cadre où elles se déroulent est spectaculaire. Une université moderne, une grande salle circulaire avec des équipements perfectionnés, une pièce d'observation avec glace sans teint. Les moments du début de l'expérience sont ellipsés pour arriver directement au stade "critique ». Après avoir assisté à une partie de l'expérience telle qu'elle se déroule pour un individu singulier, nous accompagnons le procureur Volnay dans le bureau du psychologue qui va lui exposer ses résultats, ne perdant pas totalement de vue l'enjeu fictionnel, puisqu'au passage, il nous montrera un enregistrement vidéo de l'expérience identique avec l'assassin supposé.

54 A l'issue de cette séquence de quelques minutes, le spectateur a acquis une connaissance des expériences de Stanley Milgram, presqu'aussi complète que s'il avait lu le livre «Soumission à l'autorité » qui les rapporte.

Une séquence du Serpent, film d'espionnage, consiste en un exposé en voix off, illustré par des images auxquelles on accorderait volontiers une "vérité » liée au genre « documentaire ». L'exposé porte sur l'organisation et la puissance de la C.I.A., les services d'espionnage américains. Ces deux séquences procèdent un peu différemment : dans la première, l'expérience de Milgram est reconstituée, dans un décor "photogénique » largement spectacularisé, mettant en jeu d'emblée des personnages de fiction qui nous ont été présentés antérieurement dans le film. Dans la séquence du Serpent, une véritable séquence documentaire vient interrompre le cours du récit (comme certaines descriptions perturbent momentanément l'énonciation d'un roman). Le seul élément immédiatement familier est la voix-off que le spectateur identifie facilement à celle du narrateur déjà intervenu plusieurs fois depuis le début du film. Ce n'est que vers la fin de la séquence que resurgissent des personnages de fiction, maintenant bien ancrés dans ce cadre « réel », puisqu'il nous est présenté avec les codes identifiables du documentaire. 
Mais l'intérêt de ces deux séquences tient à leur trait commun plus qu'à leurs différences. Le spectateur accède à des connaissances auxquelles un film procédant des codes « documentaires » ne serait peut-être pas parvenu à l'intéresser. Il s'agit de connaissances " austères", "scientifiques", "statistiques ». Mais dans ces deux séquences, il semble que le spectateur ne perde pas une miette des savoirs qui lui sont exposés. Cela tient sans doute à leur situation dans la progression d'un récit. Éclairant ce qui précède, possiblement nécessaires pour comprendre ce qui suit, les savoirs exposés se trouvent "valorisés» par leur intégration dans la fiction. Ce procédé garantit une attention soutenue du spectateur pendant un exposé didactique (austère) : on ne sait plus, le temps de ces séquences, si c'est la fiction qui se met au service d'un bref documentaire, ou l'inverse, si tant est que cette distinction documentaire/fiction soit pertinente, puisqu'une fois encore on se heurte à ses limites.

Une procédure singulière, relevant d'un souci également didactique, est offerte par le film de Luis Bunuel La Voie lactée. Deux personnages se rendent en pèlerinage à St Jacques de Compostelle et rencontrent divers personnages exposant leurs vues, qui toutes en leur temps, furent tenues par l'Église pour des hérésies. Traversé d'un humour jubilatoire, le film ne recule devant aucune surprise qui puisse être ménagée au spectateur, devant aucun anachronisme. Bunuel s'est expliqué un peu sur la façon dont il a procédé pour rendre attrayant l'exposé des hérésies : "Cela m'ennuyait de filmer deux prêtres en train de discuter le dogme, j'ai donc cherché une atmosphère inadéquate, pour créer une espèce de déplacement. Un maître d'hôtel, des serveurs et une serveuse qui discutent de théologie rendent la chose plus amusante que si c'étaient des cardinaux et des évêques. En outre, cet épisode est lié au fait que les pèlerins viennent d'entrer dans le restaurant pour demander l'aumône." ( Conversations avec Luis Bunuel, Cahiers du Cinéma, Paris, 1993, p. 200.)

Cette démarche, comme celle de Verneuil (avec sans doute plus d'éclat) s'appuie sur le présupposé que le spectateur s'intéresse davantage à des connaissances si elles intéressent des personnages, les concernent. Les connaissances transitent toujours sous formes de discours, ici assujettis à leurs incarnations par des agents de la fiction (les personnages).

\section{Des films « tableaux noirs »}

59 Quelques expériences cinématographiques, quoi qu'isolées (mais celles de Gance et Pollet ne le sont-elles pas?), nous incitent à évoquer un troisième type de démarche qu'on pourrait, en risquant de simplifier excessivement, présenter comme une «synthèse » des deux précédentes. C'est une démarche qui, tout en s'appuyant sur des savoirs constitués en dehors du cinéma, chercherait à organiser le film et ses émotions en fonction des discours qu'il s'approprie. Pour exemple: les films de deux collectifs de cinéma «militant » des années post-68 : Cinéthique et le Groupe Dziga Vertov ${ }^{14}$ (ce dernier, fondé par Godard). Ces deux collectifs ont fait leurs le programme du cinéaste soviétique des années 1920-30: "Voir et montrer le monde au nom de la révolution soviétique" (Dziga Vertov). C'est à nouveau comme chez Pollet, produire des films qui nous regardent avec un regard Autre (ici, le regard de la révolution), mais en même temps s'appuyer sur des discours déjà constitués. Du reste, bien souvent, ces films abordant un sujet directement ou indirectement politique (ex : la Tchécoslovaquie, les handicapés, le cinéma lui-même, telle lutte en particulier...) procèdent en déconstruisant et critiquant successivement une série de discours idéologiques caractéristiques de ceux qui les prononcent (discours 
«bourgeois", "réformistes", "gauchistes", etc.). Mais il ne s'agit pas simplement d'exposer une critique de discours constitués, mais plutôt d'enseigner comment les critiquer. Voici comment Godard formule sa démarche en 1969: «Pendant la projection d'un film impérialiste, l'écran vend la voix du patron au spectateur: la voix flatte, réprime ou matraque. Pendant la projection d'un film révisionniste, l'écran est seulement le haut-parleur d'une voix déléguée par le peuple mais qui n'est plus la voix du peuple, car le peuple regarde en silence son visage défiguré. Pendant la projection d'un film militant, l'écran est simplement un tableau noir ou un mur d'école qui offre l'analyse concrète d'une situation concrète... $»^{15}$. Un an plus tard, Godard, à la faveur d'un entretien, précise sa démarcation avec les courants dominants du cinéma militant: «Il y a deux sortes de films militants : ce que nous appelons les films 'tableaux noirs' et les films 'Internationale', celui-ci qui équivaut à chanter l'Internationale dans une manif, l'autre qui démontre et permet à quelqu'un d'appliquer dans la réalité ce qu'il vient de voir, où d'aller le récrire sur un autre tableau noir pour que d'autres puissent l'appliquer aussi $»^{16}$. L'organisation des films répond bien à ce projet: autour d'une situation (par exemple la Tchécoslovaquie de 1969 dans Pravda, le mouvement de mai 68 implicitement évoqué dans Vent d'est, etc.), le spectateur est confronté à un certain nombre de discours qui s'affrontent et se critiquent mutuellement, et il est contraint de les caractériser et de se positionner. Il s'exerce ainsi au travail politique, apprend à le pratiquer.

Plus : le film, procédant en plusieurs étapes, formule lui-même l'autocritique des modes de représentation mises en œuvre dans ses premiers moments. Pravda, film en quatre parties, montre d'abord ( $1^{\mathrm{re}}$ partie) des images de la Tchécoslovaquie, qu'un regard candide commente (en gros: "on est bien dans un pays capitaliste: il en a toutes les caractéristiques »). La $2^{\mathrm{e}}$ partie critique la démarche mise en œuvre dans la première, disant en substance : «tout ça ce n'était que des impressions de voyage...c'est de la connaissance sensible, mais ça ne suffit pas... Il faut transformer cette connaissance en connaissance rationnelle ». La deuxième partie re-sollicite des images vues dans la première en nommant, en caractérisant théoriquement et politiquement ce qu'on y voit: le révisionnisme. ( Révisionnisme, c'est-à-dire, dans le vocabulaire marxiste-léniniste, la révision du marxisme par des sociaux-démocrates, révision qui aboutit à des falsifications théoriques et des trahisons politiques: en l'occurrence, la réorientation de la transition socialiste en Tchécoslovaquie vers une politique de restauration du capitalisme. Cet emploi du terme est antérieur à l'acception actuelle pour révisionnisme historique) $3^{\mathrm{e}}$ partie : «sur une image de maladie, mettre un son qui n'est pas malade...Mettre un son juste avec une image fausse pour retrouver une image juste». C'est ainsi que la voix-off du film explique comment elle procède. Elle continue: "Première partie, on a vu la situation concrète: un pays malade. Deuxième partie, on a analysé concrètement cette situation concrète: on a cherché de quelle maladie il s'agissait. On a trouvé : cette maladie c'est le révisionnisme. » Et dans cette troisième partie, le film sollicitera à nouveau les mêmes images, organisées différemment, suivant d'autres rapports et d'autres hiérarchies, avec, en voix-off, des textes politiques (principalement issus de la Révolution Culturelle chinoise), incitant à la lutte contre le révisionnisme, sur les cadres, les paysans, l'armée, la dictature du prolétariat... La $\mathbf{4}^{\mathbf{e}}$ partie, adopte un ton qui relève davantage des mots d'ordres politiques, elle veut inciter à l'action: en gros, pour le renversement des révisionnistes qui gouvernent la Tchécoslovaquie. On me pardonnera le résumé un peu simpliste que je fais d'un film qui n'est que très rarement projeté ${ }^{17}$, je cherche à en exposer brièvement la construction qui, en soumettant au spectateur un cheminement de pensée (en l'occurrence, un cheminement matérialiste-dialectique: marxiste), veut le rendre capable de le saisir, de le 
restituer, voire de le remettre en œuvre. D'une certaine façon, la construction du film importe plus que le détail du contenu de l'analyse, (aujourd'hui défraîchi puisque la Tchécoslovaquie n'est plus socialiste et s'est divisée en deux républiques). Les discours successifs qui se trouvent critiqués ne sont pas caricaturés, tout au plus les trouve-t-on parfois concentrés en l'expression de leurs présupposés. Dans Vent d'Est, de la même façon, les discours de différents courants politiques sont successivement représentés, se critiquant les uns les autres, pour qu'à travers ce cheminement triomphe le discours révolutionnaire. C'est évidemment la nature de ce cheminement qui doit donner raison au discours révolutionnaire, à la différence des pratiques les plus répandues à l'époque dans le cinéma « militant » (terme discutable à mon sens : tout le cinéma est militant $)^{18}$.

61 De nombreux films militants, se contentaient de filmer des luttes, avec plus ou moins de soin ou de talent, dans des conditions plus ou moins touristiques. Comme si le fait d'informer sur les luttes, d'augmenter la visibilité de ceux qui les mènent, devait naturellement, mécaniquement entrainer les spectateurs à les suivre ou les imiter. C'est une conception du cinéma, identique à celle qui règne dans les appareils d'État. C'est même celle qui sert, depuis des décennies, de justification à la censure. Par exemple, avec l'argument : « il ne faut pas montrer la violence à l'écran, les jeunes l'imitent ». Avec cette parfaite hypocrisie, on censure des films pour masquer toutes les origines que peut avoir la violence dans nos sociétés. (Au hasard des nombreuses causes identifiables : qui peut en toute légalité passer la journée à traîner dans nos rues avec un pistolet à la ceinture ?...) Certains secteurs en lutte considèrent la revendication de visibilité comme un aspect de leur lutte (c'est le sens de manifestations comme la Lesbian \& Gay pride) : n'y a-t-il pas là le symptôme de la domination d'une conception qui :

1. constate et tient pour acquis le pouvoir du visible dans un monde « où l'on dit volontiers "je vois" pour " je comprends $»^{19}$; et

2. se refuse à contester cette domination du visible en demandant au contraire à y avoir sa part du gâteau.

62 Au contraire de ce despotisme du visible, le groupe Dziga Vertov, le collectif réuni autour de la revue Cinéthique, pouvaient considérer le réel comme irréductible au visible. Dans cette part invisible (inaudible, disons: non-sensible) du réel, réside, entre autres, l'organisation du visible : les règles qui le régissent (règles sociales, naturelles, etc.). Serge Daney a résumé clairement l'enjeu cinématographique de ce constat dans une critique de Comment Yukong déplaça les montagnes de Joris Ivens et Marceline Loridan : " Une caméra et un magnétophone qui se branchent naïvement sur la réalité chinoise rencontrent nécessairement cette pré-mise en scène sociale. Soit elle la reconduit (en la faisant passer pour spontanée), soit elle la fera oublier un instant (mais alors, il faut la morceler). Le naturalisme est une technique qui reconduit quelque chose qui lui préexiste: la société en tant qu'elle est déjà une mise en scène. Travailler ce donné, casser cette pré-mise en scène, la rendre visible en tant que telle est une opération courageuse, difficile, impopulaire. Le réalisme est toujours à gagner. $»^{20}$

63 Pour ces collectifs (Cinéthique, Dziga Vertov), il s'agit de penser cinématographiquement la réalité en vue de la transformer. Ce en quoi ces films appliquent le programme de la XI ${ }^{\text {ème }}$ thèse sur Feuerbach de Marx : «Les philosophes n'ont fait qu'interpréter diversement le monde, ce qui importe, c'est de le transformer ». Envisagé en conformité avec cette thèse, le cinéma devient un levier possible d'une transformation à laquelle les militants travaillent par ailleurs. Si la rencontre avec un film peut s'avérer aussi importante que celle avec un individu, le cinéma peut quelque chose. Il peut contribuer à agir pour une transformation de la société. 
64 A l'instar de ces films entretenant une relation "poétique» au monde, ceux-ci nous invitent à anticiper sur d'autres organisations possibles de la société en ne se limitant pas à "faire correspondre à un travail sans émotions des émotions sans travail " (l'expression est de Claude Bailblé). N'y a-t-il pas à travers ces voies des continents du cinéma qui restent à explorer ?

«Ambitionnant une transformation révolutionnaire de la société, cherchant à faire des films conséquents avec cette ambition, les groupes Cinéthique et Dziga Vertov tiennent parole. Conscients des processus en jeu dans la réception d'un film, ils ambitionnent, par leurs films, de réformer les représentations qu'ils savent être à l'œeuvre dans les cerveaux de leurs spectateurs. Voulant armer ces spectateurs, pour qu'eux-même puissent relayer l'expérience que leur a fait vivre le film, les collectifs intègrent dans leur démarche cinématographique la recherche de formes didactiques, pédagogiques, à l'instar de celles que les dramaturges épiques (Bertolt Brecht, Erwin Piscator, Peter Weiss, Groupov de Liège) ont su trouver au théâtre. ${{ }^{21}}^{11}$

Le cinéma peut quelque chose. En tant que moyen technique bien sûr, mais surtout en tant qu'art de la représentation: il peut s'affranchir d'une fonction de moyen de " communication de masse " à laquelle l'ont assujetti des nécessités de reproduction de l'ordre social. Des précédents, comme des encouragements, nous montrent que le cinéma peut nous aider à nous libérer des prisons qui se bâtissent silencieusement dans nos têtes, avec le ciment des déjà-vus, déjà-connus et autres stéréotypes.

Connaissances, croyances, idéologies trouvent en nous des logements tantôt voisins, tantôt identiques, sans que l'on soit en mesure d'établir une discrimination entre elles. Sur cette base d'appui, les cinémas dominants, ceux de l'éternel retour du même (même scénarios, mêmes visages et silhouettes de vedettes, mêmes relations aux appareils techniques qui constituent le cinéma, ...) assurent tranquillement dans nos moments de loisirs (c'est-à-dire de reproduction de la force de travail) la reproduction de l'ordre (du désordre) social tel qu'il est.

Néanmoins, si c'est cette pratique concourant à la reproduction de l'ordre social qui domine au cinéma, on peut trouver divers exemples de démarches qui échappent à cette servitude : des démarches didactiques qui, au sein du cinéma dominant, font accéder le spectateur à des savoirs utiles, constitués hors du cinéma. Mais aussi, à travers une relation poétique au monde, des démarches qui produisent un nouveau mode sensible d'acquisition des connaissances, spécifiquement cinématographique, permettant de saisir des connaissances nouvelles.

Une synthèse dialectique de ces deux types de démarches a pu donner lieu à des démarches de type "tableau noir ", renouant avec une conception brechtienne ${ }^{22}$ qui voudrait voir les effets de l'œuvre se prolonger consciemment dans la vie. Consciemment, car il apparaît que toute œuvre produit des effets dans la vie: pour aller vite, en renforçant (reproduction) ou en fragilisant (transformation) des représentations déjà ancrées dans le cerveau du spectateur. Ainsi, ceux qui veulent voir dans les représentations artistiques un reflet de la réalité, devront admettre que ces représentations agissent, en sont un reflet actif, puisqu'elles changent quelque chose (en reproduisant-renforçant ou en fragilisant-transformant) les représentations sur lesquelles les sujets s'appuient pour orienter leurs propres actions dans la vie. 


\section{NOTES}

1. Le présent texte s'inscrit dans une recherche en cours, dans le cadre d'une Thèse de cinéma sous la direction de Gérard Leblanc, Université de Paris III / Censier.

2. Octave Mannoni, Clefs pour l'imaginaire ou l'Autre scène, Seuil, Paris, 1969, p. 14.

3. On peut d'ailleurs ajouter que "la folie ", en tant qu'écart aux comportements socialement tolérés, s'engendre, parfois, d'une déconnexion de la réalité chez un sujet resté comme suspendu dans un imaginaire d'évasion, plus confortable pour supporter l'existence. Le sujet alimente son utopie intérieure et se complaît dans l'illusion d'y vivre et d'y évoluer « réellement ».

4. Voir note 3.

5. Pour de plus amples développements sur cette question, voir : «Le théâtre du point de vue de l'imaginaire ", in Octave Mannoni, Un si vif étonnement (La honte, le rire, la mort), Seuil, Paris, 1988, pp. 13-37.

6. Dominique Lecourt (Une crise et son enjeu, François Maspéro, coll. Théorie, Paris, 1973.) conteste le terme de "théorie de la connaissance", auquel il propose d'opposer des "thèses pour la connaissance». Bien qu'en accord avec cette position, je continue d'employer le terme de «théorie de la connaissance », pour ne pas alourdir le présent texte avec ce débat.

7. C'est le singulier qui appelle les guillemets.

8. Il s'agit du chapitre III de son ouvrage Les Vérités de La Palice, François Maspéro, Paris, 1975.

9. Ce constat est partagé par Gérard Leblanc, «L'utopie gancienne », in 1895, n 31, octobre 2000 «Abel Gance : nouveaux regards », voir en particulier la p. 95, entièrement consacrée à la place des oiseaux dans Cyrano et d'Artagnan.

10. Gérard Leblanc, «Du déplacement des modalités de contrôle », Hermès, $n^{\circ} 25$ : «Le dispositif, entre usage et concept », CNRS Editions, Paris, décembre 1999.

11. On pourra s'en convaincre par la lecture d'un petit livre qui nettoie la tête sur un mode jubilatoire : Relevé provisoire de nos griefs contre le despotisme de la vitesse, à l'occasion de l'extension des lignes du TGV (1991), Editions de l'Encyclopédie des Nuisances, Paris, 1998.

12. Gérard Leblanc, "presque une conception du monde...", in Après Deleuze (Philosophie et esthétique du cinéma), Dis Voir, Paris, 1996, p. 146.

13. Yves Bonnefoy, «La poétique de Nerval» in La Vérité de parole et autres essais, Mercure de France, Paris, 1988 (folio-gallimard, p. 49). (C'est moi qui souligne ; ce texte est cité par G. Leblanc dans « presque une conception du monde ", op. cit.)

14. Le groupe a adopté le nom de Dziga Vertov, en référence à ce cinéaste soviétique (1896-1954) concepteur du «ciné-œil» et autres formules polémiques. Documentariste, il attribuait au montage la fonction de rendre visible l'organisation du réel ( du point de vue de la révolution prolétarienne »). Encore faut-il préciser que le montage commençait pour lui dès la prise de vue, puisqu'on y sélectionne déjà ce qu'on va montrer...

15. Cf. Godard par Godard, éditions de l'Etoile - Cahiers du cinéma, Paris, 1985, p. 338.

16. Cf. Godard par Godard, éditions de l'Etoile - Cahiers du cinéma, Paris, 1985, p. 348.

17. On trouvera néanmoins le texte de la bande son de la plupart des films du groupe Dziga Vertov dans les Cahiers du Cinéma, $n^{\circ} 238-239$ et 240 (dans ce dernier $n^{\circ}$, en particulier le texte de Pravda), et des analyses plus détaillées de ces films dans les mêmes numéros, ainsi que dans : Jean Collet \& Jean-Paul Fargier, Jean-Luc Godard, Seghers, coll. "Cinéma d'aujourd'hui », Paris, 1974. (Attention! Les films du groupe Dziga Vertov ne sont évoqués que dans cette édition et pas dans les éditions antérieures, parues sous la seule signature de Jean Collet) - Voir aussi : Gérard 
Leblanc: * «Quelle avant-garde?»(Cinéthique $\left.n^{\circ} 7-8\right) ; *$ "Sur trois films du groupe Dziga Vertov » $\left(\mathrm{VH} 101, n^{\circ} 6\right) ;^{*}$ «Lutte idéologique et Lutte en Italie » (VH 101, $\left.n^{\circ} 9\right)$.

18. Le cinéma «militant» n'existe pas. En tout cas pas si on le conçoit comme un cinéma s'opposant à un autre qui, bien entendu, ne serait pas «militant». Cinéma «militant»: la formule est insuffisante dans la mesure où tout le cinéma l'est. Reste à savoir « militant »... pour (ou contre) quoi ? Le cinéma nazi, n'est-il pas « militant »? Le cinéma industriel, la publicité en tête, ne sont-ils pas des cinémas «militants » du patronat ? Il est donc impossible de parler de cinéma « militant » sans dire pour quelle orientation ce cinéma milite. Impossible de penser une articulation entre cinéma et politique indépendamment des orientations politiques et des conceptions du monde qui se trouvent matérialisées par les films. La remarque vaut aussi bien pour d'autres formules rapides et réductrices équivalentes comme : « cinéma politique », etc.

19. La formule est de Serge Daney. Cet usage sémantique n'est pas une exclusivité du Français.

20. Serge Daney, La Rampe, Cahiers du Cinéma - Gallimard, Paris, 1996, p. 70 (c'est l'auteur qui souligne).

21. Si le documentaire est depuis bien longtemps constitué comme genre cinématographique, les expériences de théâtre documentaire restent fort marginales. Il ne nous reste que quelques écrits, photos et témoignages du travail de Piscator. Un peu plus pour Peter Weiss, dont certains textes de théâtre sont traduits en français, en particulier, pour ceux relevant directement du théâtre documentaire: L'Instruction (réédité à L'Arche) et Discours sur la guerre du Vietnam (précédé d'une "Note sur le théâtre documentaire»), au Seuil. Mais l'exemple le plus contemporain et le plus convainquant est le travail réalisé par la troupe du Groupov de Liège, sur le génocide des Tutsi rwandais («Rwanda $1994 »)$. (Contact Groupov : 26-28, rue Bois l'Evêque, B-4000 Liège, tél. : (00) (32) [0]-4-253-61-23).

22. Voir par exemple (et parmi tant d'autres) le bref développement dans : Bertolt Brecht, Sur le réalisme, L'Arche, Paris, 1970, p. 159 : « Pas de réaliste en art qui ne soit réaliste hors de l'art ».

\section{AUTEUR}

\section{DAVID FAROULT}

Docteur en Cinéma, cinéaste, chargé de cours à l'Université Paris VIII 